\title{
Automatisation des mesures sur carottes de sondage de la densité du bois, de son retrait et des contraintes de croissance
}

\author{
J.R. PERRIN et J.Ch. FERRAND * \\ I.N.R.A., Station de Recherches sur la Qualité des Bois \\ Centre de Recherches forestières, Champenoux, F 54280 Seichamps \\ H.N.R.A., Station d'Amélioration des Arbres forestiers \\ Centre de Recherches forestières d'Orléans, Ardon, F 45160 Olivet
}

\begin{abstract}
Résumé
Nous présentons deux appareils de mesure entièrement automatiques imaginés et réalisés à la Station de Recherches sur la Qualité des Bois, qui utilisent la technique des microprocesseurs.

Le premier appareil consiste en une automatisation intégrale d'un microdensitomètre pour la mesure de la densité du bois d'après un négatif de radiographie. On décrit les principes retenus pour les parties mécanique, électronique câblée et logicielle de l'appareil. Le choix des dimensions de la fente de mesure est discuté et illustré par des exemples, et l'étalonnage est présenté en détail. On donne également des exemples de résultats et des indications sur la durée des mesures qui est récluite.

Le deuxième appareil permet de mesurer au micron près et simultanément les diamètres axial et tangentiel au long d'une carotte de sondage qui se déplace sur un chariot. Les detix applications principales sont l'estimation des retraits (tangentiel et axial) et des contraintes de croissance. Cet appareil est décrit avec les mêmes détails que le précédent.
\end{abstract}

\section{Introduction}

Depuis plus de 20 ans, la $4^{*}$ Section de la Station de Recherches et Expériences de l'Ecole Nationale des Eaux et Forêts, puis la Station de Recherches sur la Qualité des Bois ont développé des méthodes de mesure des propriétés du bois sur carottes de sondage : infradensité, microdensitométrie sur négatif radiographique (PoLGE, 1963), diamètres des carottes pour le calcul des retraits tangentiel et longitudinal (PoLgE et al., 1973) et des contraintes de croissance (Polge \& Thiercelin, 1979; Ferrand, 1982 a), rendement en fibres et qualités papetières (JANIN, 1972), vitesse des ultrasons pour lévaluation des propriétés élastiques du bois (Bucur, 1981), etc.

$V_{u}$ l'intérêt des résultats obtenus, il est apparu indispensable d'automatiser les plus longues de ces opérations, afin d'augmenter la capacité de mesure.

Cet article présente deux réalisations : l'automatisation intégrale d'un microdensitomètre, et celle d'un appareil à mesurer les diamètres des carottes. Toutes deux 
utilisent un microprocesseur dont elles constituent une application simple et relativement peu coûteuse.

\section{Mesure de la densité du bois d'après un négatif de radiographie}

La mesure de la densité du bois d'une carotte de sondage sur un négatif de radiographie (Polge, 1963) a ouvert des horizons nouveaux pour l'étude de la qualité des bois et de ses sources de variation.

Cependant, les services rendus par cette méthode ont toujours été limités par deux facteurs : la relative lenteur des mesures et le fait que la densité ne fait généralement pas partie des propriétés recherchées par les utilisateurs du bois, même si elle est corrélée avec nombre d'entre elles (Polge, 1966).

A l'origine, on effectuait un dépouillement manuel de la courbe tracée par le microdensitomètre; ensuite, KELler \& XeuXet (1973) ont proposé une méthode semi-automatique incluant une table codeuse $(X, Y)$ pour la saisie et la perforation sur ruban de papier des données brutes et un programme écrit en Fortran pour l'obtention des résultats (densités minimale, maximale, moyenne, largeur de cernes, etc.).

Nous présentons ici un appareillage original, conçu et réalisé à la Station de Recherches sur la Qualité des Bois, qui constitue une automatisation intégrale de la mesure à partir du négatif de radiographie. Cet appareillage utilise principalement un microdensitomètre Joyce $3 \mathrm{CS}$ et un microprocesseur Thomson-Efcis 6800 .

\subsection{Description}

La conception de l'appareil laisse le choix entre de nombreuses configurations qui dépendent des besoins et des objectifs de l'utilisateur. La figure 1 représente un système très complet correspondant à des besoins de recherche. Les unités indispensables en routine y sont encadrées dun double trait. Il s'agit de :

1) le densitomètre lui-même,

2) une adaptation débrayable pour le déplacement de la table de mesure par un moteur pas à pas (1) dont la vitesse est réglable,

3) un dispositif de «transformation en densité » délivrant une tension de $1 \mathrm{mV}$ par $\mathrm{kg} / \mathrm{m}^{3}( \pm 1$ p. 100),

4) un convertisseur analogique-numérique (12 bits),

5) un pupitre de commande et l'électronique associée,

6) le microprocesseur et une unité de calcul arithmétique,

7) un périphérique de sortie.

Pour une configuration plus évoluée, on peut ajouter :

8) un enregistreur pour tracer la courbe de variation de la densité (1),

(1) Quand la table de mesure est entrainée par le dispositif (2), la table d'enregistrement est mise hors service, d'où l'utilité de l'enregistreur $n " 8$. 
9) 12 compteurs, montés en parallèle, pour le décompte du nombre de mesures dépassant des niveaux de densité programmables,

10) un circuit adaptant la vitesse de mesure à la variation instantanée du signal,

11) des périphériques supplémentaires.

\subsection{Fonctionnement}

Le fonctionnement est entièrement automatisé. L'opérateur met le négatif sur la table de mesure et effectue l'étalonnage. Ensuite, il place le début d'un échantillon sous la fenêtre de mesure, indique les paramètres de mesure, et lance l'exécution, qui s'arrêtera d'elle-même après le nombre de cernes souhaité.

Voici quelques détails sur ces opérations :

\subsection{Etalonnage}

Les radiographies sont réalisées comme sur la figure 2. On ajoute aux échantillons et au coin optique radiographique (1) une cale d'étalonnage en acétate de cellulose comportant deux zones : l'une est faite de trois paliers (2) d'épaisseur connue $(0,48,1,99,4,00 \mathrm{~mm})$, l'autre d'un prisme (3) dont l'épaisseur varie linéairement de 0,35 à $5 \mathrm{~mm}$ environ. L'image du coin optique radiographique est placée dans le faisceau optique de référence (fig. 3). On analyse alors l'image du prisme (3) en agissant sur le coin manuel (4) jusqu'à ce qu'on obtienne une droite (fig. 4 A). On trace ensuite la courbe correspondant aux paliers (2) et on trace des droites correspondant au niveau moyen de chacun de ces paliers (fig. 4 B). Connaissant l'épaisseur des échantillons à mesurer, on calcule la densité équivalente de chaque palier, et l'on choisit les deux mieux adaptés à la gamme des densités à mesurer.

Ce choix étant fait, il reste à régler les potentiomètres agissant sur l'origine et le gain du circuit de «transformation en densité » $\left(n^{\circ} 3\right.$ au paragraphe 1). Pour cela, on ramène le palier bas sous le faisceau de mesure et on règle l'origine, puis on se place sur le palier haut pour régler le gain. Il suffit de recommencer cette manœuvre environ trois fois pour obtenir la stabilité de l'affichage. L'étalonnage est terminé.

\subsection{Fonctionnement de l'appareil}

L'appareil se déplace d'un pas de mesure (i), s'arrête, prend la valeur de densité, la convertit en numérique (soit $y_{i}$ ), la transfère au microprocesseur qui teste l'hypothèse «fin de cerne»; si la réponse est négative, il traite cette valeur et recommence le cycle jusqu'à une fin de cerne. Celle-ci est détectée lorsque :

1) on a rencontré au moins un minimum local de densité depuis le début du cerne en cours,

2) la variation locale de densité est négative et supérieure en valeur absolue à une valeur donnée (programmable),

3) la valeur de la densité est plus proche du minimum que du maximum du cerne en cours,

[4) sur option, la machine demande confirmation à l'opérateur]. 


Selon le programme utilisé, on peut transférer chaque valeur de densité sur ruban de papier (ou tout autre support) pour traitement ultérieur sur ordinateur, ou bien calculer à chaque étape $\mathrm{i}$ les valeurs $\Sigma y_{\mathrm{j}}$ et $\Sigma \mathrm{y}_{\mathrm{i}}{ }^{2}$; dans ce cas, on sort en fin de cerne les résultats suivants : la largeur du cerne, les densités moyenne, minimale et maximale, et l'écart-type de la densité qui sert de critère d'hétérogénéité du bois (FERRAND, 1982 d). On trouve sur la figure $5 \mathrm{~A}$ un exemple correspondant à la première option (impression de toutes les valeurs ponctuelles de densité, au pas de mesure choisi, pour un accroissement annuel), et sur la figure $5 \mathrm{~B}$, une illustration de la deuxième possibilité : sortie des seules données synthétiques pour 10 cernes de l'échantillon de douglas de la figure 2 .

I1 faut ajouter que l'appareil offre bien d'autres possibilités, par l'extension des programmes (logiciel) et par l'utilisation plus complète de la logique câblée [compteurs de niveaux (9) $\$ 1.1$, de la capacité mémoire et des périphériques.

\subsection{Dimensions de la fenêtre de mesure et du pas de mesure}

Les aspects théoriques du choix des dimensions de la fenêtre de mesure ont déjà été présentés (FERRAND, 1982 c). Une illustration pratique des conséquences de ce choix est donnée sur la figure 4 où sont comparées les courbes d'étalonnage obtenues avec les fenêtres de $0,05 \times 0,10 \mathrm{~mm}=A$ [proche des $0,05 \times 0,06 \mathrm{~mm}$ employée par Parker et al. (1980)] et $0,025 \times 1,00 \mathrm{~mm}=\mathbf{B}$ [couramment utilisée à la Station

\section{Fig. 1}

$\mathrm{a}$ et $\mathrm{b}$ : Appareillage automatique pour la mesure de la densité du bois d'après un négatif radiologique.

$\mathrm{a}$ and $\mathrm{b}$ : Automatic device for wool density measurements on radiographs.

1 : Microdensitomètre Joyce 3 CS.

Joyce 3 CS microdensitometer.

2 : Adaptation débrayable pour le déplacement de la table de mesure. Disengageable mechanism driving the measurement table.

3 : Dispositif de «transformation en densité » (amplificateur). Analogic amplifier for density.

4: Convertisseur analogique-numérique. Analogic digital converter.

5 : Pupitre de commande. Command desk.

6: Microprocesseur (a) et unité de calcul arithmétique (b). Microprocessor (a) and arithmetic computing unit $(b)$.

7 : Périphérique (ici télétype et perforateur de ruban). Teletype + paper tape puncher.

8 : Enregistreur. One way plotter.

9 : Compteurs. Latch of individuals counters.

10: Asservissement de la vitesse de mesure. Monitoring of measurement speed.

11 : Périphériques supplémentaires. Peripherals. 


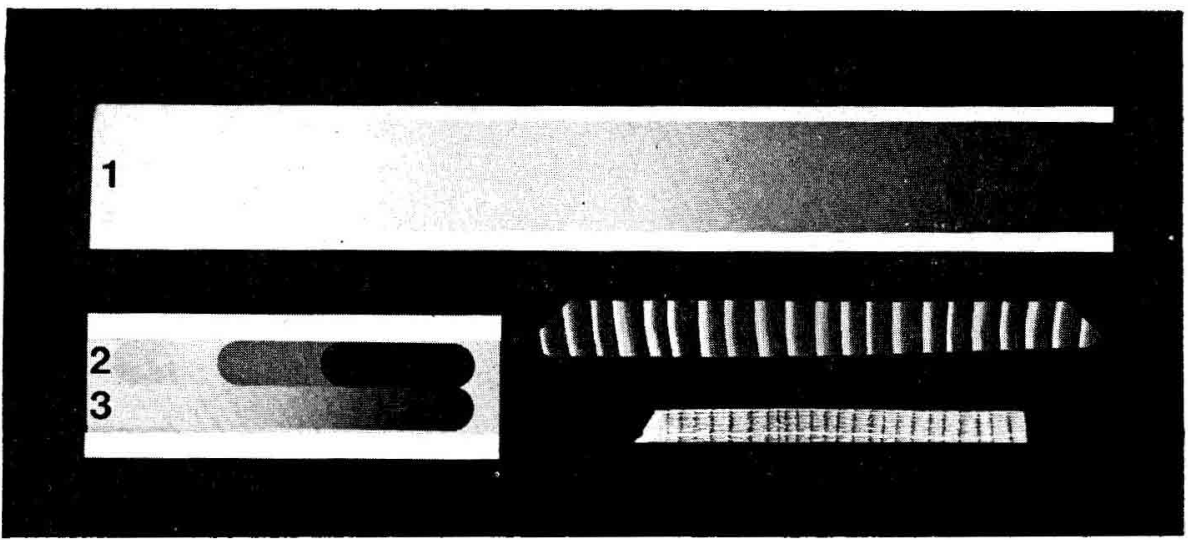

FIG. 2

Image d'un négatif radiographiqụe, avec un échantillon de chêne et un de douglas.

Radiographic film (negative) with an oak sample and a douglas fir one.

1: Coin optique.

Optical wedge.

2 : Cale à 3 paliers.

3 steps gauge.

3 : Prisme (coin utilisé pour l'étalonnage).

Prismatic wedge for calibration.

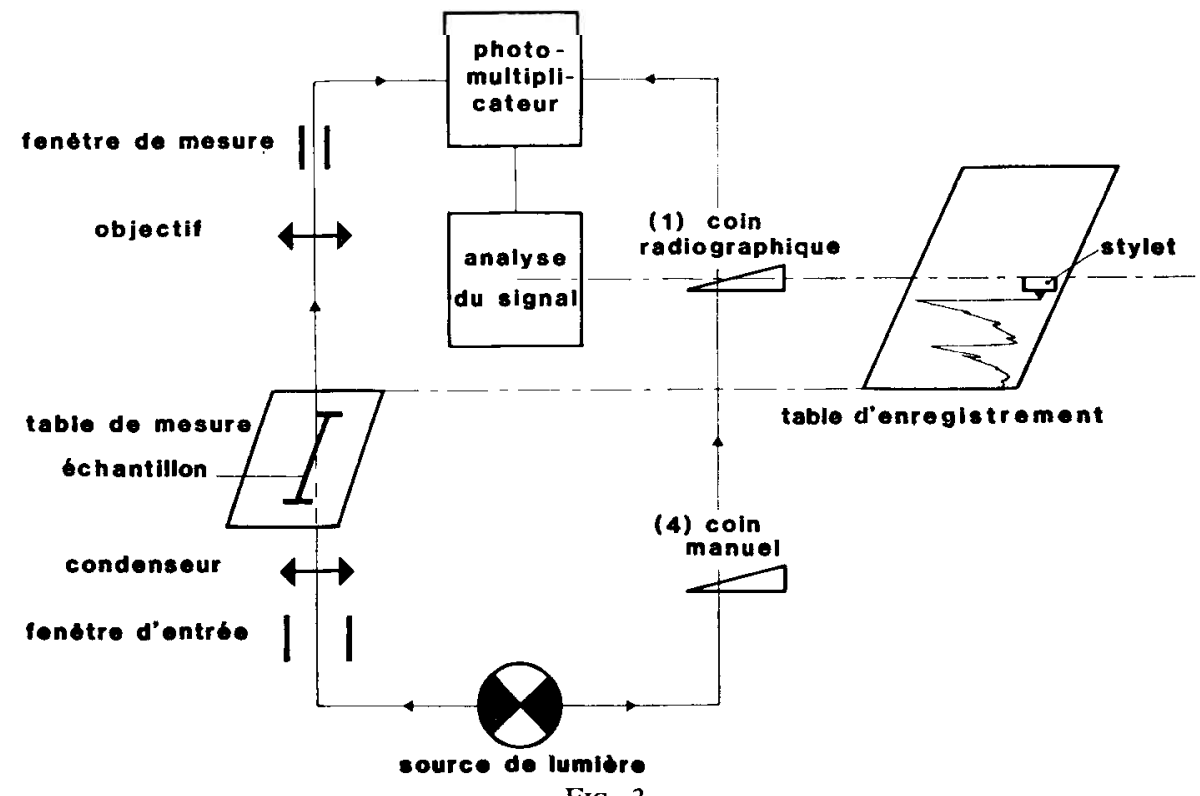

FIG. 3

Schéma simplifié du microdensitomètre, permettant de comprendre les opérations d'étalonnage.

Simplified diagram of the densitometer, which helps for understanding the calibration process. See text. 


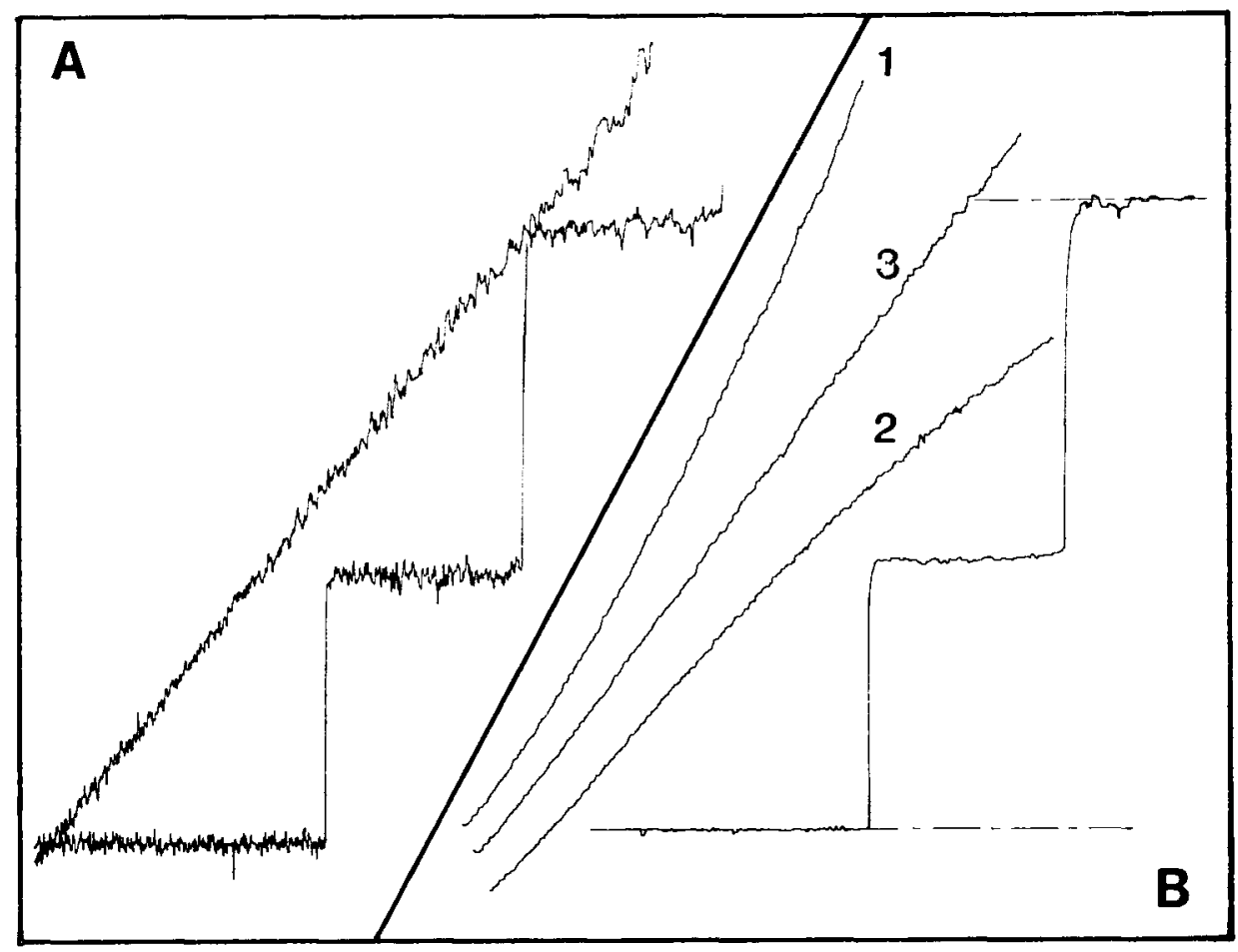

Fici, 4

Calibration du densitomitre; courbes obtenues avec le prisme (n" 3, fig. 2) et la cale à 3 paliers ( $n^{\prime \prime} 2$, Jig. 2).

Calibration; curves obtained with the prismatic wedge $(n " 3, f i s .2)$ and with the 3 steps gauge ( $n^{\prime \prime} 2$, fig. 2).

A : Avec une lenêtre de mesure de $0,05 \times 0,10 \mathrm{~mm}$. Le bruit de fond est considérable. Dimensions of the measurement shit : $0.05 \mathrm{~mm} \times 0.10 \mathrm{~mm}$. Noise is very large.

B : Avec une fenêtre de mesure de $0,025 \times 1,00 \mathrm{~mm}$.

Dimensions of the measurement slit : $0.025 \mathrm{~mm} \times 1.00 \mathrm{~mm}$.

1 et 2 : Etapes du réglage du coin manuel (n" 4, fig. 3).

Stages of adjustmemt of the mannal nedge ( $n^{\prime \prime} 4$, fig. 3).

3 : Obtention d'une réponse linéaire.

A linear response.

de Recherches sur la Qualité des Bois, et plus proche de celle de $0,030 \times 1,60 \mathrm{~mm}$ proposée par Schwfingruber et al. (1978)]. Il est indispensable de choisir une grande dimension tangentielle de la fente si l'on veut éviter le bruit de fond dû entre autres au grain du film radiographique. De même, lors de la mesure d'un échantillon, la fenêtre de type A intercepte alternativement plus de parois ou plus de lumens et donne un aspect erratique aux variations de densité, même dans le cas d'un conifère comme le douglas (fig. $5 \mathrm{C}$ ). Avec une fenêtre de type $\mathrm{B}$, les variations sont plus 


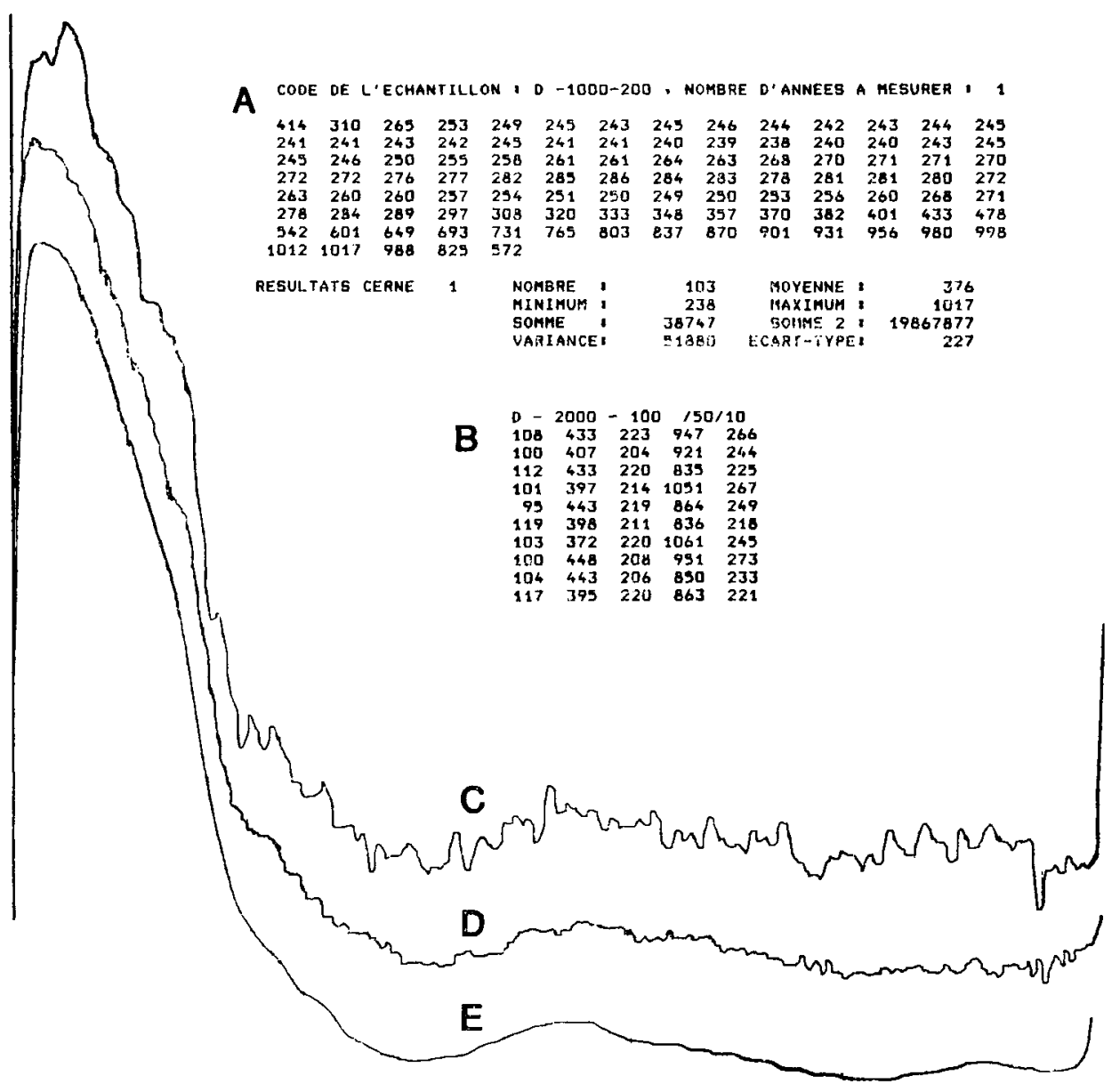

Fic; 5

Exemples de documents obtenus en sortie a partir de léchantillon de douglas de la figure 2. Examples of the outputs obtained rith the douglas fir sample of figure 2.

1. Listirgs délivrés par le télétype $\left(r^{\prime *} 7\right.$, fig. 1):

Listings of the teletype (n" 7 , fig. 1$)$ :

A : Programme imprimant les valeurs individuelles de densité (yi dans le texte) puis les résultats des calculs.

With the program printing all the measured values of densiry (y in the text). and the results of calculations.

B : Programme imprimant seulement les résultats des calculs (code échantillon/pas de mesure/nombre de cernes à mesurer. Puis, pour chaque cerne, dans cet ordre : nombre de pas de mesure, densités moyenne. minimale, maximale et écart-type de la (ensité).

With the program printing only the results of the calculations [sample number/measurements interval/namber of rings to be measured. Then, for each ring : number of measurement steps $(=$ ring width), mean, minimum. maximm density and standard deviation of density].

2. Courbes délivrées par l'enregistreur (n" 8, fig. 1) (pour le même cerne de la figure 5 A): Curves recorded tsee $n^{\circ} 8$. lig. 1), Jor the same ring analysed in fig. 5 , depending of he necastroment shit dimensions:

C: Fenêtre de mesure de $005 \times 005 \mathrm{~mm}$. Mecisurement slit : $0.05 \mathrm{~mm} \times 0.06 \mathrm{~mm}$.

D : Fenêtre de mesure de $0.025 \times 1.00 \mathrm{~mm}$. Measurement slit : $0.025 \mathrm{~mm} \times 1.00 \mathrm{~mm}$.

$\mathrm{E}:$ Fenêtre de mesure de $0,10 \times 2,00 \mathrm{~mm}$. Measurement slit : $0.10 \mathrm{~mm} \times 2.00 \mathrm{~mm}$. 
régulières (fig. $5 \mathrm{D}$ ) et représentent au mieux le phénomène physique; quant à la très grande fenêtre utilisée par Cown \& Clément (1983), elle donne un lissag̣e très prononcé (fig. $5 \mathrm{E}$ ).

Il faut aussi se poser la question du choix du pas ce mesure $P$ en fonction de la dimension radiale $R$ de la fenêtre de mesure. II y a trois solutions :

- si $\mathrm{P}>\mathrm{R}$, on procède à un échantillonnage à l'intérieur du cerne ;

- si $P=R$, on décrit tout le cerne, chaque point étant pris en compte une seule fois :

- si $\mathrm{P}<\mathrm{R}$, on décrit tout le cerne de façon redondante.

Parker (1980) se place dans la situation 3 avec $R=5 \mathrm{P}$. A notre sens, cela ne présente pas d'avantage particulier et multiplic la duréc des mesures par cinq pour une même valeur de $\mathrm{R}$ par rapport au cas $\mathrm{n} " 2$ choisi par les auteurs. Le seul avantage éventuel serait d'obtenir un certain lissage, mais cela devient superfłu dès que les dimensions de la fenêtre de mesure sont suffisantes. Il faudrait même se poser la question de savoir si le choix du cas $n^{\prime \prime} 1$ ne permettrait pas de gagner du temps sans trop perdre de précision. Plusieurs auteurs européens travaillent déjà de cette façon (InterJaboratory Standardization Survey, 1982).

Une solution presque identique peut être trouvée en réalisant l'égalité de $\mathbf{P}$ et de $\mathrm{R}$, mais à une valeur bien supérieure, comme le fait Cown (1983).

En conclusion, il apparaît donc qu'un large choix est laissé à l'utilisateur en fonction des caractéristiques du bois étudié (épaisscur et courbure des cernes), les points importants étant de conserver unc grande dimension tangentielle à la fenêtre dc mesure et d'adapter l'unc à l'autre la dimension radiale de cette dernière et la valeur du pas de mesure.

\subsection{Discussion}

L'intérêt de lautomatisation des mesures est triple. On peut augmenter :

- la yuantité de données et de résultats relative à un mêne échantillon,

- la rapidité de la mesure,

- sa précision.

Dans le cas de l'appareil présenté ici, le bilan est très satisfaisant. En effet, la quantité de données recueillies n'est limitée que par des facteurs indépendants de l'appareil lui-même (grain des films radios, dimension des cellules du bois, etc.; voir FERrand, $1982 \mathrm{c}$ ); ensuite, le nombre de caractéristiques que l'on retiendra pour chaque cerne ne dépend que de la capacité de calcul dont on dispose. Or celle-ci est justement très élevée grâce à la présence de l'unité de calcul arithmétique.

Le temps nécessaire à la mesure d'un échantillon avec le programme de la figure $5 \mathrm{~B}$ est d'environ 40 secondes par cerne de 3 à $4 \mathrm{~mm}$ de large avec un pas de mesure de $25 \mu \mathrm{m}$. Ce temps inclut le calcul et la sortie des résultats. Il faut ajouter 5 à 10 minutes pour l'étalonnage de la radio (qui porte 15 à 50 échantillons) et 1 à 3 minutes pour la mise en place de chaque échantillon sous la fenêtre de mesure. Finalement, il faut donc environ une heure pour 10 échantillons comme celui de la figure $5 \mathrm{~B}$. La durée de ces manipulations peut être réduite par un opératcur bien 
entraîné. Quant à la durée de là mesure elle-même, elle peut être optimisée par l'utilisation du circuit $\mathrm{n}^{\prime \prime} 10$ (\$ 1 ) qui accélère automatiquement la eadence des mesures dans les zones à faible gradient de densité.

La précision est largenent améliorée par rapport aux méthodes semi-automatiques antérieures. La répétabilité de la mesure est elle aussi excellente. Cependant, l'accroissement de précision n’est réel qu“à condition de se placer par ailleurs dans de bonnes conditions expérimentales. Outre la nécessité de bien choisir les dimensions de fenêtre de mesure, il devient impératif de ne pas travailler sur carotte de sondage, mais sur une barrette d'épaisseur constante sciée dans la carotte (THIERCELIN \& PERRIN, 1972 : Perrin, 1983).

Cela permet également d'améliorer la définition de la densité maximale et la reconnaissance des limites de cernes, pour deux raisons : les flous sont diminués et on peut travailler le long d'un trajet vraiment radial, même si la carotte n'a pas été prélevée strictement dans cette direction. Une amélioration supplémentaire serait possible dans ce domaine; il sagil de l'utilisation d'une fenêtre de mesure tournante qui permettrail de sadapter aux variations dexcentricité des limites de cerne. Ce dispositif devrait cependant être commandé à la main, ce qui risquerait de ralentir la mesure. Il serait spécialement utile dans le cas des essences à limites de cernes irrégulières (la plupart des feuillus en particulier). Une autre application serait de placer les barrettes toutes parallèles sur to négatif grâce à un gabarit, puis dutiliser la fente tournante (au lieu des réglages de la table de mesure) avant la mesure de chaque échantillon.

D'autre part, on peut se poser la question de lintérêt d’un nouvel apparcil de mesure automatique de la densité, alors que plusicurs laboratoires utilisent déjà des systèmes très évolués (EVERTSEN, 1981 ; PARKER ('f al., 1980). Il faut remarquer que l'appareil décrit ici présente des différences significatives avec les précédents:

- il est beaucoup moins cher.

- le temps nécessaire à la mesure d’un échantillon est très bref. Cela est dû à un ensemble de choix techniques : la logique de commande est câblée, la conversion analogique-numérique est effectuéc par un convertisseur (et non par un voltmètre), le microprocesseur est programmé en langage assembleur, les calculs sont effectués par une unité arithmétique spécialisée travaillant sur 16 ou 32 bits, etc.,

- il calcule et sort les résultats sans l'intervention d'un ordinateur. Cela n'cmpêche pas que lon puisse transférer les mesures brutes et/ou les résultats des calculs à un ordinateur. soit directement. soit par l'intermédiaire d’un support (magnétique, papier, etc.),

- le déplacement de la table de mesure est assuré de manière autonome par le moteur pas à pas ( $n^{\prime 2} 2$, \$ 1.1); cela évite d'avoir à mesurer en plusieurs fois les échantillons de grande longueur comme on doit le faire dans lutilisation normale du microdensitomètre. De plus, l'enregistreur ( $n^{\prime \prime} 8, \$ 1.1$ ) est asservi au déplacement de la table de mesure. La courbe obtenue reflète done fidèlement la mesure analogique (c'est-à-dire le signal avant toute transformation).

Dans lensemble, lappareil de la Station de Recherches sur la Qualité des Bois apparaît donc comme moins coûteux et au moins aussi performant que les précédents, même si le logiciel peut encore être renforcé. La conception la plus voisine se rencontre en fait chez Cown \& Clement (1983), à partir d'une technologie de mesure toute différente cependant puisquills utilisent une source radioactive qui irradic directement les barrettes de bois. 


\section{Mesure simultanée des diamètres tangentiel et longitudinal au long d'une carotte de sondage}

La mesure des diamètres des carottes de sondage a dabord fourni des informations sur le retrait du bois (par différence entre les états saturé et sec à l’air). Par la suite, dans le cas de plusieurs espèces, on a également relié la valeur du diamètre tangentiel saturé à lintensité des contraintes de croissance. La valeur de diamètre prise en compte est toujours la moyenne d’au moins 80 mesures réalisées à intervalles réguliers le long de la carotte, dans une zone choisie par lexpérimentateur.

Les deux premières versions de l'appareil de mesurc sont représentées sur la figure 6. La deuxième version était déjà automatique et utilisait un captcur à franges de moiré précis au micron près. Cependant, il ne mesurait le diamètre que clans une scule direction à la fois. Cela obligeait à manipuler la carotte avant la dcuxième mesure, et augmentait les risques de variation d'humidité, en particulier à l'état saturé. Le temps de mise en place et de mesure dans les deux directions était de 3 à 5 minutes.

Un autre dispositif unidirectionnel, légèrement différent, a été proposé par Chichignoud \& Sales (1981).

Nous présentons ici une version plus élaborée qui réalise les mesures dans les deux directions à la fois et qu: est entièrement pilotée par un microprocesseur ThomsonEfcis 6800 .

\subsection{Description}

La figure 7 présente un synoptique de l'appareil. Celui-ci comporte principalement un ensemble mécanique pour la mesure et un ensemble électronique pour la commande et le traitement de linformation.

\subsection{Parties mécaniques et de mesure}

La partie mécanique est à gauche sur la figure $7 \mathrm{~B}$. Elle comporte :

- un bâti rigide (1) qui porte les éléments suivants,

- une vis à billes (2) actionnée par un moteur pas à pas (3),

billes (2),

- un chariot (4) qui se déplace sur deux guides (5) sous laction de la vis à

- le chariot (4) pousse la carotte par l'intermédiaire d'un mandrin (6) libre de ses mouvements (pour les cinq degrés de liberté non concernés par la translation du chariot),

- deux potences (7) [sur lesquelles sont fixés les capteurs (10)] et deux contretouches (8), toutes quatre réglables ( 3 trans'ations, une rotation),

[- rautomatisation du relevage (9) des touches de mesure est en cours].

Les touches de mesure et les contre-touches (8) entrent en contact avec l'échantillon le long d'une génératrice d'un cylindre de carbure de tungstène de $1 \mathrm{~mm}$ de diamètre et $4 \mathrm{~mm}$ de long. 


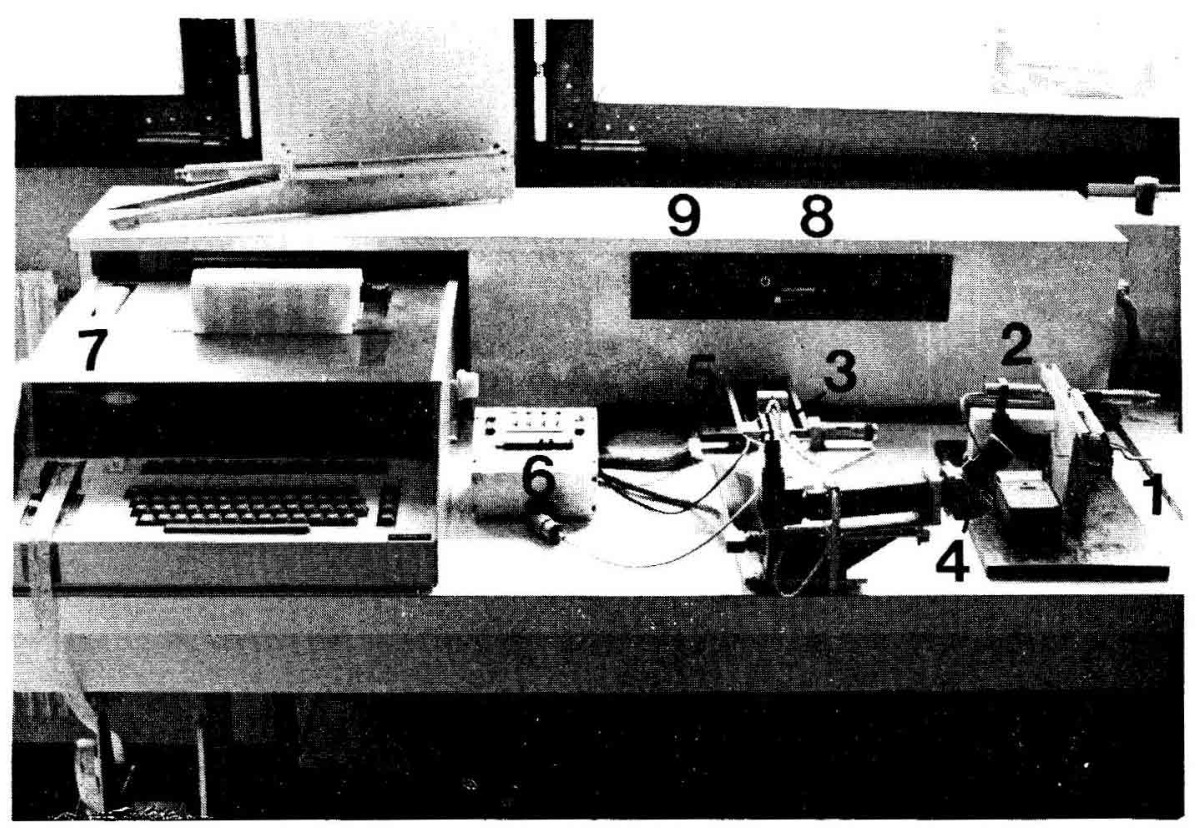

FIC. 6

Versions antérieures des appareils à mesurer les diamètres des carottes.

Earlier devices for increment core diameter measurements.

- A droite : première version, manuelle :

On the right : first device, manual :

1: Manivelle commandant l'avancement de la carotte.

Crank for sample displacement.

2: Micromètre à transformateur différenticl, à $0,01 \mathrm{~mm}$.

Differential transformer micrometer. Accuracy $0.01 \mathrm{~mm}$.

- A gauche : deuxième version automatique :

On the left : second device, automated :

3 : Bâti mécanique.

Frame.

4: Lunette de visée télescopique.

Optical adjustment viewer.

5 : Micromètre ì franges de moiré, à $0,001 \mathrm{~mm}$.

Moire fringes micrometer. Accuracy $0.001 \mathrm{~mm}$.

6 : Pupitre de commande. Command desk.

7 : Télétype et perforateur de ruban. Teletype and paper tape puncher.

8 : Affichage de la valeur courante. Sampled value display.

9 : Aflichage de la moyenne courante. Mean value display. 
Les capteurs sont des micromètres digitaux de précision qui utilisent la méthode des franges de moiré : une poutre en verre est solidaire de la tige de mesure et comporte dans sa fibre neutre une graduation de $10 \mu \mathrm{m}$ en $10 \mathrm{fm}$. Cette poutre se déplace devant une autre règle de verre fixe qui porte une graduation formant vernier avec la précédente. Il en résulte des franges de moiré qui sont transformées en impulsions par une cellule photosensible. Les impulsions sont traitées par un compteur (11) qui fournit la dimension mesurée sous forme numérique.

\subsection{Ensemble électronique}

La partie électronique comprend (fig. 7) :

(11) le microprocesseur et une unité de calcul arithmétique,

(12) un pupitre de commande,

(13) les deux compteurs solidaires des capteurs,

(14) une interface,

(15) une imprimante.

\subsection{Fonctionnement}

Contrairement au cas du microdensitomètre, la configuration est ici entièrement figée, et le mode opératoire est unique. Le programme a été fixé sur mémoire morte reprogrammable et réalise le cycle de fonctionnement représenté sur la figure $8 \mathrm{~A}$.

L'opérateur refève les touches des capteurs, met en place la carotte, affiche sur le pupitre de commande l'identité de l'échantillon, la longueur à mesurer et le pas de mesure (les pas autorisés sont de $0,1 \mathrm{~mm}$ à $3,9 \mathrm{~mm}$ par dixième et les longueurs de $0,1 \mathrm{~mm}$ à $250 \mathrm{~mm}$ par dixième). Il lance alors l'exécution en enfonçant la touche «mesure» du pupitre. Un fac-similé des sorties est donné sur la figure $8 \mathrm{~B}$. Une légère modification du programme permettrait d'ajouter des sorties supplémentaires telles que celle des valeurs individuelles, des minima, maxima, écarts-types des deux diamètres.

Le temps nécessaire à la mesure d'une zone de $25 \mathrm{~mm}$ par pas de $0,25 \mathrm{~mm}$ (100 pas de mesure) est d'environ 2 minutes et 30 secondes tout compris.

\subsection{Discussion}

Cet appareil est dune conception bien différente de celle du microdensitomètre. Il ne sag:ssait en effet pas d'automatiser un appareil du commerce, mais bien de fabriquer un instrument de mesure complet et automatique. L'ensemble mécanique a représenté une grosse partie du travail. Il faut noter le soin qui a été apporté dans la conception du mandrin (6) et dans celle des réglages des potences (7) et des contretouches $(8)(\$ 2.11)$ afin de rendre impossibles de mauvais positionnements de la carotte et des touches de mesure qui introduiraient des erreurs expérimentales.

Au contraire, la partie électronique est un peu plus légère. Le microprocesseur utilise directement l'état des fonctions du pupitre de commande pour décicer de la prochaine opération à effectuer. L'interface sert principalement de sélecteur, en entrée comme en sortie. Dans le cas du microdensitomètre au contraire, la logique câblée du pupitre de commande commandait tout le processus jusqu'au moment où une valeur de densité était prête à être lue. Le microprocesseur était alors sollicité pour effectuer les calculs ; sitôt ceux-ci terminés, il rendait le contrôle à la logique câblée qui cffectuait le pas de mesure suivant, etc. 

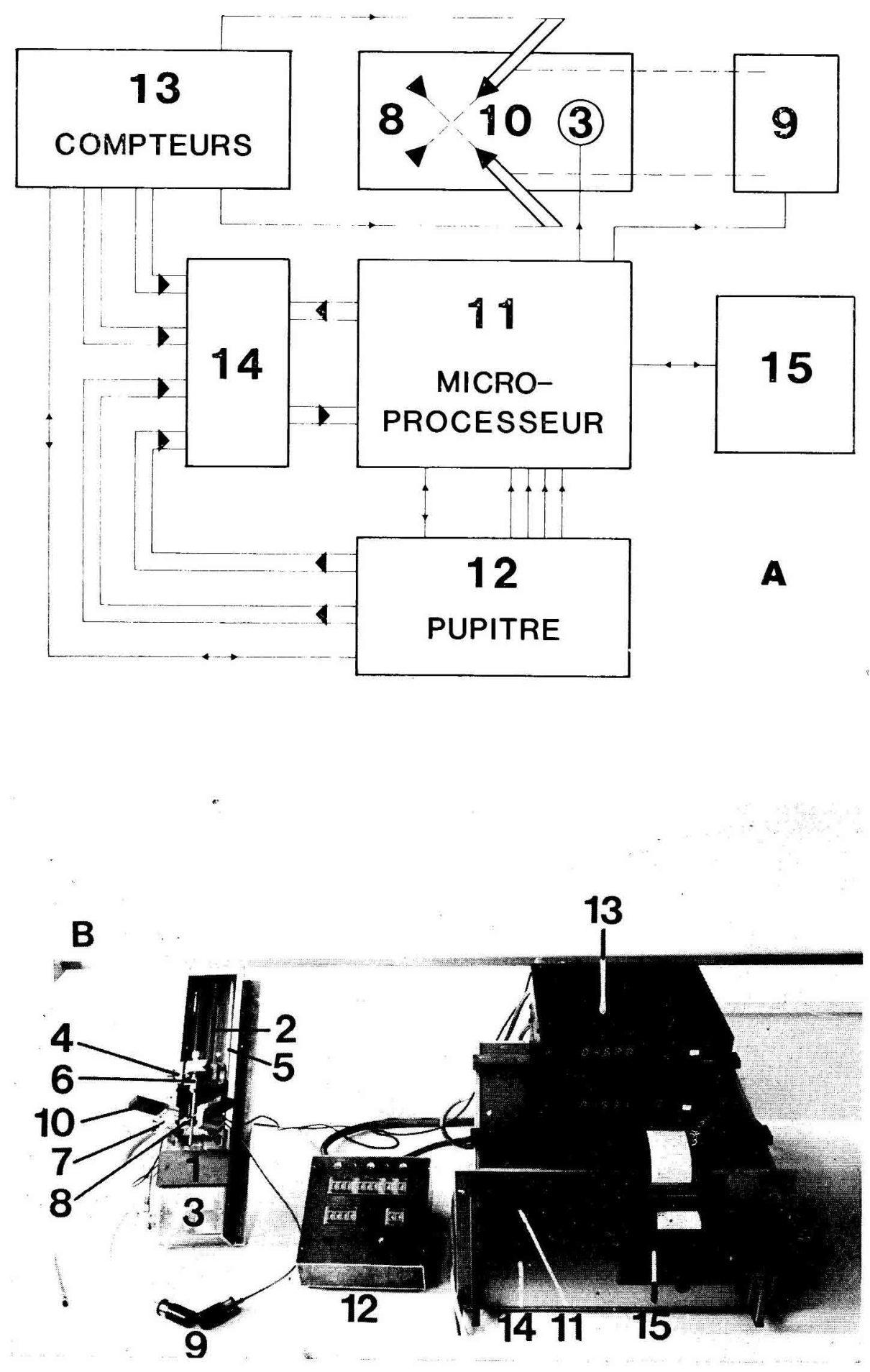
Cet appareil de mesure des diamètres a été conçu pour être complémentaire à celui de la figure 6. En effet, les avantages de la mesure simultanée dans les deux directions s'accompagnent d'une certaine rigidité : on ne peut mesurer que des carottes de $5 \mathrm{~mm}$ de diamètre. C'est pourquoi l'appareil de la figure 6, qui permet de mesurer des échantillons de toutes formes jusqu'à une épaisseur de $10 \mathrm{~mm}$, sera conservé et même revu pour accepter une dimension maximale de $50 \mathrm{~mm}$. L'ensemble des deux machines permettra de réaliser dans les directions tangentielle et longitudinale toutes les mesures de dimension sur échantillon long dont a besoin la Station de Recherches sur la Qualité des Bois.

Firi. 7

Nonvelle version de lappareil à mesurer les diamètres des carontes.

New increment core diameters measurement device.

A : Synoptique de la partic électronique.

Diagram of the electronic part.

B : Vue d'ensemble :

General view. See text :

1 : Bâti mécanique.

Frame.

2: Vis à billes. Lecul scren.

3 : Moteur pas à pas. Stepping motor.

4: Charriot. Carriage.

5 : Guide. Rails-guide.

6: Mandrin. Shaft .

7 : Potences. Supports:

8: Contre-touches. Fixed jan's.

9: Relevage des touches de mesure. Lifting of measurement jaws.

10 : Micromètres à franges de moiré à $0,001 \mathrm{~mm}$. Micrometer (moire fringes).

11: Microprocesseur et unité de calcul arithmétique. Microprocessor and A.C.U.

12 : Pupitre de commande. Command desk.

13 : Compteurs. Counters.

14 : Interface. Interface.

15 : Imprimante. Printer. 


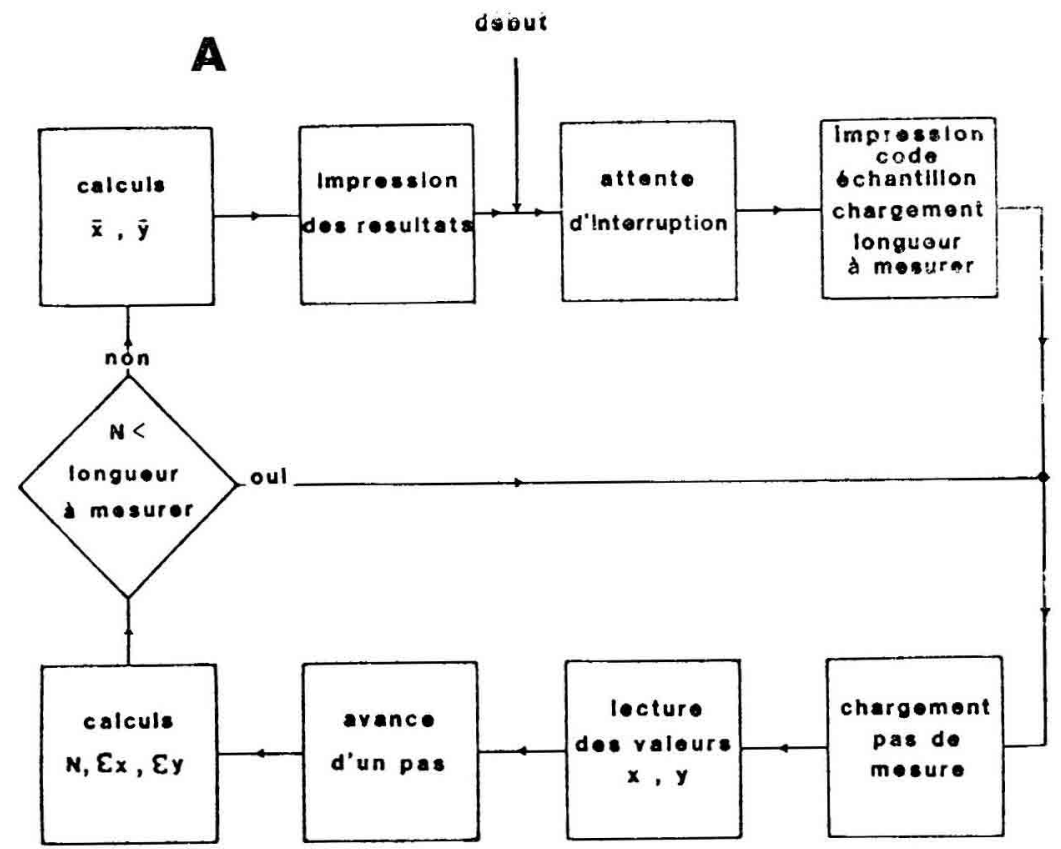

$427-812-2-2$

NOMPRE : ?RG

B

DIAMETRE THNRENTIEL

$\begin{array}{lr}\text { SUMIME : } & 973281 \\ \text { MOYENNE : } & \text { Q8EF. }\end{array}$

DIPIAETRE RXIHL.

$\begin{array}{lr}\text { SOMME : } & 1061559 \\ \text { AOYENNE : } & 5.398\end{array}$

Fig. 8

Programmation de l'appareil à mesurer les diamètres des carottes.

Programing the cores measurement device.
A : Lc programme actuel.
Aciual program.
B : Les sorties correspondantes.
Corresponding outputs. 


\section{Conclusion}

On a souligné deux facteurs limitants pour rutilisation fructueuse des mesures densitométriques : la lenteur des mesures et le peu d'intérêt des utilisateurs du bois pour la densité en elle-même. Nous pensons que l'automatisation présentée ici est à même de repousser très loin la première limite et, dans une certaine mesure, la seconde car la puissance et la précision de mesure accrues permettront de mettre en lumière de nouvelles raisons de s'intéresser à la densité du bois. De plus, le coût modéré et la simplicité de cette réalisation la rendent accessible à un nombre plus élevé de laboratoires.

De même, l'automatisation rend plus rapide et plus fiable la mesure des diamètres des carottes de sondage. La réalisation de grandes séries de mesures a déjà permis d'acquérir des connaissances décisives sur la variabilité des contraintes de croissance et sur celle du retrait chez le hêtre (Ferrand, 1982 b; Polge, 1981).

L'utilisation des microprocesseurs permettra de réaliser les mêmes progrès dans de nombreux domaines, au laboratoire comme sur le terrain, et augmentera en quantité et en qualité les possibilités de la recherche forestière.

\section{Summary \\ Fully automated devices for measurement of wood density, shrinkage and growth strains on increment cores}

We present two fully automated measurement devices, designed and built at the Station de Recherches stur la Qualité des Bois in Nancy, France, and using the techniques of microprocessors.

The first device is a full automation of a microdensitometer and allows the measurement of wood density from a radiographic film. One describes the principles used for building in the mechanical, hardware and software parts of the device. The question of measurement slit dimensions is discussed and illustrated with examples. Calibration is explained in details. Output examples are shown and measurement times figures are given, which prove to be significantly reduced.

The second device allows increment cores diameters measurements along an increment core; the accuracy is $0.001 \mathrm{~mm}$ and both axial and tangential diameters are measured at the same time. The main applications are shrinkage and growth strains measurements. This device is described in the same details than the precedent one.

\section{Références bibliographiques}

ANONYME, 1982. Interlaboratory standardization survey. Bulletin de liaison de la microdensitométrie du bois, 2 (2), octobre 1982.

Bucur Voichita, 1981. Détermination du module d'Young du bois par une méthode dynamique sur carottes de sondage. Ann. Sci. For., 38 (2), 283-298.

Chichignoud Michèle, Sales Ch., 1981. Contribution à l'étude de la qualité des bois de plantation de Grevillea robusta, origine République du Burundi. Document du Centre Technique Forestier Tropical, Division Essais et Emplois des Bois, Nogentsur-Marne, mars. 
Cown D.J., Clement B.C.. 1983. A wood densitometer using direct scanning with X-rays. Wond Sci. Terhnol. 17 (2), 91-99.

Evertsen J.A., 1981. Computerised integration of datit acquisition and processing. Wood Microdensitometry Bulletin, rol. 1, n" 3, 6-12.

Ferrand J.Ch., 1982 a. Etude des contraintes de croissance. 1 - Méthode de mesure sur carottes de sondage. Ann. Sci. For., 39 (2). 109-142.

FtrRAND J.Ch., 1982 b. Etude des contraintes de croissance. 2 - Variabilité en forêt des contraintes de croissance du hêtre (Fagus sylvaica L.). Ann. Sci. For., 39 (3), 187-218.

Ferrand J.Ch., 1982 c. Réflexions sur la clensité clu bois. 1 - Délinition de la densité du bois. Holzforschung, 36 (2), 99-105.

Ferrand J.Ch., 1982 d. Réflexions sur la densité du bois. 2 - Calcul de la densité et de son hétérogénéité. Holzforschung. 36 13). 153-157.

JANIN G., 1972. Microcuissons papetières. Méthode adaptéc aux recherches forestières portant sur la détermination des calactéristiques papetières individuelles sur arbres vivants à l'aide d'échantillons de bois dont le mode de prélèvement, l'aspect et le poids ne sont pas usuels. La papeterie, n" 3, mars.

Keileer R., XeUXet D. 1973. Méthode de la mesure des données microdensitométriques et de leur traitement à l'ordinateur. Communication présentée au Congrès de l'I.U.F.R.O., Le Cap, Prétoria, sept-oct.

PARKer M.L., Bruce R.D., Jozsa L.A., 1980. X-rity densitometry of wood at the Western Forest Products Laboratory. Technical Report. n" 10.

Perrin J.R., 1983. Dispositif de sciage de carottes de sondage de $5 \mathrm{~mm}$ sans collage préalable sur un support. Amn. Sci. For.. 40 (4), 399-406.

Polge H., 1963. Une nouvelle méthode de détermination de la texture du bois : l'analyse densitométrique de clichés radiographiques. Ann. Ec. Natl. Eaux Forêts Stn. Réch. Exp., XX (4), $531-581$.

POLge H., 1966. Etablissement des courbes de variation de la densité du bois par exploration densitométrique de radiographies d'échantillons prélevés à la tarière sur des arbres vivants. Applications dans les domaines technologique ef pluysiologique. Thèse Doct. Scie. Appli., F. Université Nancy I.

Polge H., Keller R., Thitrcelin F., 1973. Inlluence de l'élagage de branches vivantes sur la structure des accroissements annuels et sur quelques caractéristiques du bois de Douglas et de Grandis. Ann. Sci. For., 30 (2). 127-140.

Polge H., Thiercelin F., 1979. Growth stress appraisal through increment core measurements. Wood Sci., 12 (2), 86-92.

Polgi: H., 1981. Influence des Élaircies sur le's contraintes de croissance du hêtrc. Ann. Sci. For., 38 (4), 425-447.

Schweingruber F.H., Fritts H.C., Bräker O.U., Drew L.G.. Schär E., 1978. The X-ray technique as applicd to dendroclimatology. Tree-Ring Bulletin, 38, 61-91.

Thierscelin F., Perrin J.R., 1972. Débit d'échantillons d'épaisseur rigoureusement uniforme à l'aide de scies-fraises jumelées. Ann. Sci. For., 30 (2), 295-299. 